\title{
Optimal maintenance strategy for systems with two failure modes
}

\author{
Rui Peng ${ }^{\mathrm{a}}$, Bin Liu ${ }^{\mathrm{b}}$, Qingqing Zhai ${ }^{*}$, Wenbin Wang ${ }^{\mathrm{a}, \mathrm{d}}$, \\ ${ }^{a}$ Donlinks School of Economics and Management, University of Science and Technology Beijing, \\ Beijing 100083, China. \\ ${ }^{b}$ Department of Systems Engineering \& Engineering Management, City University of Hong Kong, \\ Kowloon, Hong Kong. \\ ${ }^{c}$ Department of Industrial and Systems Engineering, National University of Singapore, Singapore. \\ ${ }^{d}$ Faculty of Business and Law, Manchester Metropolitan University, UK. \\ ${ }^{*}$ Corresponding author \\ Phone: $+65-94678450$ \\ Email: zhaiqing59@126.com
}

\begin{abstract}
This paper considers a single-unit system subject to two types of failures: a traditional catastrophic failure and a two-stage delayed failure. Periodic inspections are carried out to identify the defective stage of the two-stage failure process, whereas preventive replacements are implemented to avoid any potential failure due to the catastrophic failure mode. We construct a basic maintenance model and then extend it to the cases of imperfect inspections (i.e., inspections that do not always notice a defective state). We analyze the renewal process of the system and establish the expected long-run cost rate (ELRCR). The optimal inspection period and preventive replacement interval are determined by minimizing the ELRCR. A case study on infusion pumps is presented to illustrate the proposed model.
\end{abstract}

Keywords: periodic inspection; preventive replacement; delay-time; two-stage failure process; imperfect inspection. 


\section{Introduction}

With the ongoing development of technology, modern systems are becoming increasingly complicated and often have components that are subject to multiple failure modes (Wang \& Wu, 2014). In general, these failure modes can be classified into two categories: hard failures and soft failures. Hard failures are the failures whose occurrence is instantaneous and, most likely, self-announcing. Soft failures, on the contrary, would generate early warning signals or have degradation patterns, which may be detected by inspection or monitoring.

Modern complex systems, e.g., micro electromechanical systems (MEMS) and complex medical devices, are usually subject to both hard failures and soft failures (Park et al., 2013 and Peng et al., 2010). For instance, MEMS contain both mechanical and electrical parts. The mechanical parts suffer wear that may be monitored or inspected, whereas the electrical parts may fail suddenly and make inspections fruitless for preventive maintenance. For complex medical devices such as infusion pumps, hard failures can occur due to the malfunction of the alarm and circuit breaker/fuse, while soft failures occur on the labeling and battery/charger. The voltage of the battery is routinely checked, whereas the circuit breaker/fuse cannot be monitored and may therefore fail suddenly.

To maintain a high availability, inspection is a commonly applied technique for modern plant systems (Mendes et al., 2014 and Taghipour \& Banjevic, 2012). Through inspection, potential defects can be identified and preventive maintenance actions can be carried out (Nguyen et al., 2015 and Wu et al., 2016). Accordingly, system failure can be avoided and the operational cost of the system can be reduced. Thus, it is an effective measure to improve the quality and performance of the system. Inspections can be conducted periodically (Biswajit \& Saren, 2016, Yang \& Jae, 2014 and Liu et al., 2016a), on a condition-based basis (Dieulle et al., 2003 and Michele et al., 2015), 
or after the completion of successive tasks (Zhao \& Nakagawa, 2013 and Liu et al., 2016b). Periodic inspection is widely adopted in practice due to its easy implementation and effectiveness. Wang (2009) formulated an inspection model with two kinds of periodic inspection: minor inspection and major inspection. Aven and Castro (2009) studied the optimal periodic inspection policy under safety constraints. Instead of considering cost as the single objective, Ferreira et al. (2009) investigated the optimal inspection interval in a multi-criteria framework.

Inspections are effective only if there are defective states for the system, so that the system can be repaired preventively before a failure occurs. This leads to the usefulness of the delay-time concept. Originally proposed by Christer (1976), the delay-time concept regards the failure mechanism as a two-stage process, where the first stage is from the installation to the point of a defect's arrival and the second stage (known as the delay-time stage) is from the start of the defective state to the failure, if left unattended. This concept has inspired many subsequent studies, such as Christer (1999), Wang (2012) and Zhao et al. (2015).Williams and Hirani (1997) studied the optimal inspection policy for multi-state systems with multi-level maintenance based on the delay-time model. Christer and Lee (2000) modified the delay-time model by considering the downtime caused by failures. Wang (2011) extended the traditional two-stage delay-time model to a three-stage process and studied the associated optimal inspection policy. An overview of the recent delay-time-based maintenance models can be seen in Wang (2012).

In practice, inspections can be imperfect due to the limitation of detection techniques and the effect of environmental variations. An inspection may fail to identify a defective state or mistakenly treat a normal state as the defective state (Biswajit \& Saren, 2016, Flage, 2014 and Phan \& Zhu, 2015). Usually the performance of an inspection is measured in terms of the probability of defect detection and the probability of a false alarm (Sahraoui et al., 2013). Berrade et al. (2012) formulated an imperfect 
inspection model and investigated the reliability of a system with a defective state. The imperfect inspection model was further extended to the scenario of a finite horizon (Berrade et al., 2013). Sheils et al. (2010) developed a two-stage inspection policy to assess deteriorating infrastructure, in which the detection process is divided into two stages: inspection and sizing. Mohammadi et al. (2015) integrated the imperfect inspection model into a manufacturing system, where the optimal production period and inspection policy were obtained. One limitation of the previous studies is that they only consider one failure mode. In reality, a system is usually subject to multiple failure modes (Liu et al., 2013 and Park et al., 2013). Imperfect inspection for a system with multiple failure modes requires more investigation.

In this paper, we study the maintenance policy for a single-unit system subject to two different failure modes. Failure mode 1 is the soft failure, and the failure process is formulated using the delay-time model. Periodic inspections are conducted to detect the possible defective state of failure mode 1. The case of imperfect inspection is also considered by assuming that the probability of detection is constant. For failure mode 2, which belongs to the hard failure, the failure rate increases with the system age. Preventive replacement is implemented to renew the system so as to decrease the system failure rate. Appropriate preventive replacement policy is appreciated to balance the failure probability and maintenance cost. To reduce the operational cost, the optimal inspection and preventive replacement intervals that minimize the expected long-run cost rate (ELRCR) are studied.

The remainder of this paper is organized as follows. Section 2 gives the detailed system description and assumptions. Section 3 studies the basic model by assuming that the inspection is perfect and the preventive replacement interval is an integer multiple of the inspection interval. Different renewal scenarios are investigated in detail, and the expected renewal cycle length together with the expected renewal cycle cost based on the renewal process are formulated. Section 4 extends the basic model to the case with 
imperfect inspections. Section 5 illustrates the proposed inspection and preventive maintenance model with a case study on infusion pumps. Section 6 concludes the paper and discusses possible directions for future study.

\section{Notations}

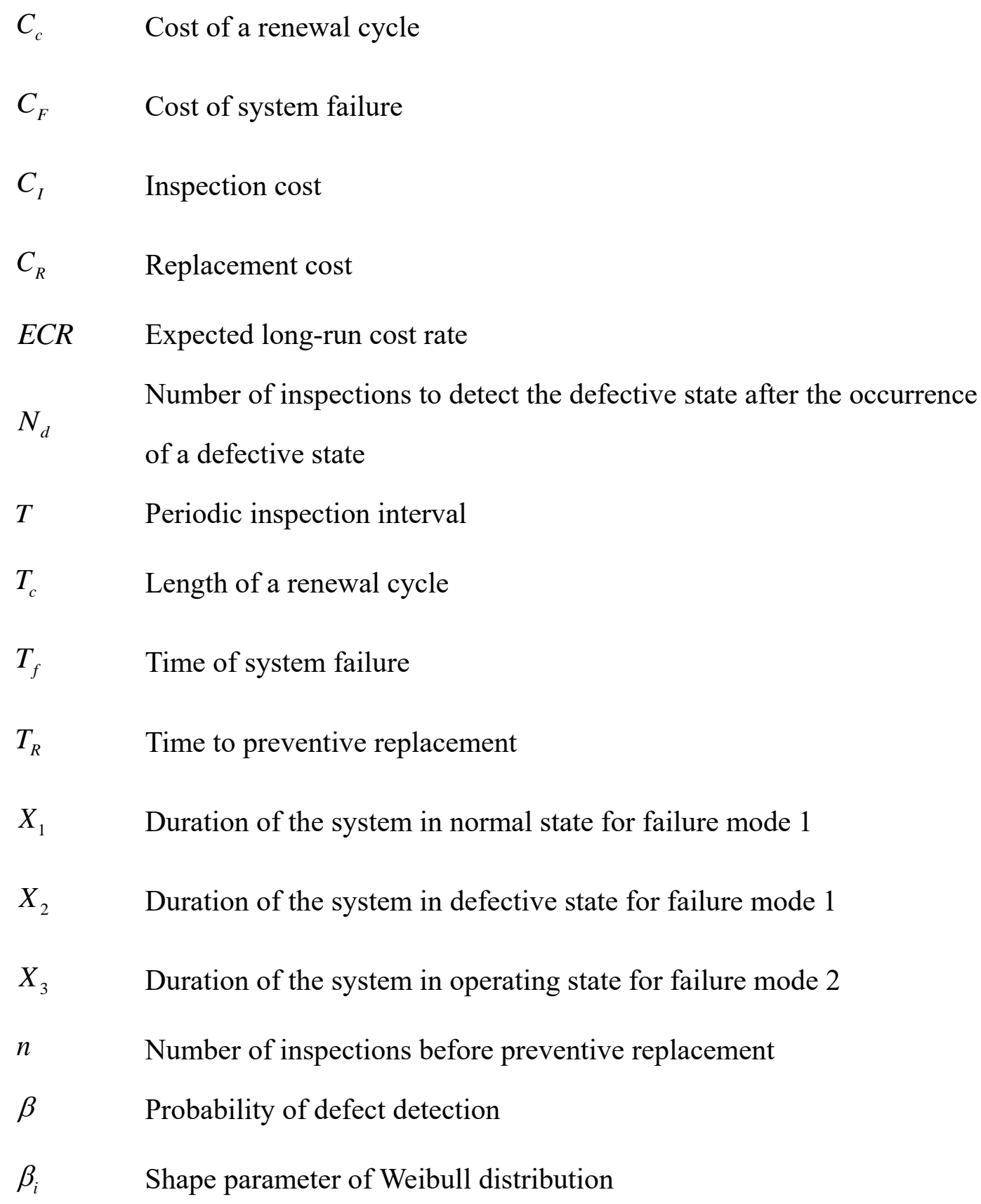




\section{System description}

The system under consideration is a single-unit system subject to two different, independent failure modes. Both failure modes lead to the system failure. Failure mode 1 consists of a two-stage failure process, which is modeled using the delay-time concept. With respect to mode 1 , the system is first in the normal state, and then experiences a defective stage prior to the eventual failure. The durations of the normal state and the defective state are described by two independent random variables $X_{1}$ and $X_{2}$, respectively. Denote the corresponding cumulative distribution functions (CDFs) as $F_{i}(\cdot), i=1,2$, and the corresponding probability density functions (PDFs) as $f_{i}(\cdot)$.

Failure mode 2 corresponds to a hard failure, i.e., the failure occurs without any prior warning, either because the defective state cannot be identified or there is no delay time at all. The random time before failure for mode 2 is denoted by $X_{3}$. The corresponding $\mathrm{CDF}$ and PDF are $F_{3}(\cdot)$ and $f_{3}(\cdot)$, respectively.

To detect the possible defective state of failure mode 1, a periodic inspection of period $T$ is carried out during the operation of the system. The probability of defect detection $\beta$ is assumed to be a constant (Williams \& Hirani, 1997). Whenever the defective state of failure mode 1 is detected, the system is immediately replaced. Since failure mode 2 has no defective stage, inspection is ineffective. Nevertheless, to mitigate the system failure due to mode 2 , a preventive replacement is carried out at $T_{R}$ if no defective stage has been identified before $T_{R}$. Whenever a failure occurs, it can always be detected, and the system can be immediately renewed. Compared with the operation period of the system, the time taken to inspect and renew the system is 
assumed to be negligible.

Let $C_{I}$ be the cost of a single inspection. We assume the cost of replacement at inspections is equal to the preventive replacement cost at $T_{R}$, which is denoted as $C_{R}$. When a failure occurs, corrective replacement is implemented to remedy the consequences of the failure with the associated cost $C_{F}$. Clearly, $C_{F}$ should satisfy $C_{F}>C_{R}$. The cost items due to failure mode 1 and failure mode 2 are identical.

\section{The basic model}

Before establishing the maintenance model, we investigate the stochastic behavior of the system subject to two failure modes.

Consider failure mode 1 first. We have the CDF of failure mode 1 as

$$
F_{12}(t)=P\left(X_{1}+X_{2}<t\right)=P\left(X_{1}<t, X_{2}<t-X_{1}\right)=\int_{0}^{t} f_{1}(\tau) F_{2}(t-\tau) d \tau .
$$

As failure mode 1 and failure mode 2 are independent, the CDF of the system lifetime $T_{f} \quad$ can be obtained as

$$
\begin{aligned}
F(t) & =P\left(T_{f}<t\right)=P\left(X_{1}+X_{2}<t \cup X_{3}<t\right)=1-\left(1-F_{12}(t)\right)\left(1-F_{3}(t)\right) \\
& =F_{12}(t)+F_{3}(t)-F_{12}(t) F_{3}(t) .
\end{aligned}
$$

With this result, we can proceed to formulate the maintenance model and further analyze the effectiveness of the maintenance policy.

In this section, we assume that the inspection is perfect $(\beta=1)$ and that the preventive replacement interval is an integer multiple of the inspection interval, i.e., $T_{R}=n T$, for integers $n \geq 1$. The system can be renewed in the following cases: (1) the defective state of failure mode 1 is detected at the $k$ th inspection, $k=1, \ldots,(n-1) ;(2)$ a preventive replacement is carried out at $n T$; and (3) failure occurs between $((k-1) T, k T), k=1, \ldots, n$. 
In the following, we first analyze these three possible scenarios and derive their corresponding probabilities. Denote $T_{c}$ as the length of a renewal cycle, and $C_{c}$ as the cost in a renewal cycle. The expected renewal cycle length $E\left(T_{c}\right)$ and the expected renewal cost $E\left(C_{c}\right)$ can be obtained subsequently. Finally, the ELRCR, which is a function of $T$ and $n$, can be obtained as (Do et al, 2015; Wu et al, 2016)

$$
E C R(T, n)=\frac{E\left(C_{c}\right)}{E\left(T_{c}\right)}
$$

Once the ELRCR is obtained, the optimal maintenance policy $\left(T^{*}, n^{*}\right)$ that minimizes $\operatorname{ECR}(T, n)$ can be obtained easily with numerical methods.

\subsection{Analysis of the renewal scenarios}

The occurrence of renewal case (1) indicates that the system is still in the normal state at $(k-1) T$, that a defect occurs before $k T$, and that neither failure mode 1 nor failure mode 2 occur before $k T$, as illustrated in Fig. 1. Hence, the probability of this scenario is

$$
\begin{aligned}
P\left(T_{c}=k T\right) & =\operatorname{Pr}\left\{(k-1) T<X_{1}<k T, X_{1}+X_{2}>k T\right\} \operatorname{Pr}\left\{X_{3}>k T\right\} \\
& =R_{3}(k T) \int_{(k-1) T}^{k T} f_{1}(t) R_{2}(k T-t) d t, k=1, \ldots, n-1 .
\end{aligned}
$$

where $T_{c}$ is the length of a renewal cycle and $R_{i}(\cdot)=1-F_{i}(\cdot), i=1,2,3$ denotes the reliability function of the normal state, defective state and failure mode 2 , respectively. 


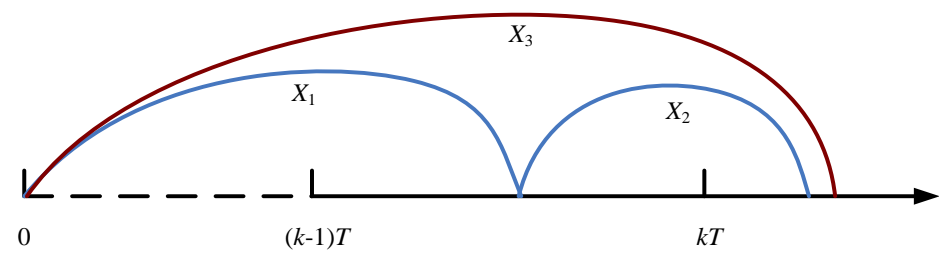

Fig.1 Renewal of the system due to the detected defective state of failure mode 1 .

If no defective state is detected in the first $(n-1)$ inspections, the system has to be renewed at $n T$ given no failure occurs before $n T$. This case indicates that the system due to failure mode 1 is still in the normal state at $(n-1) T$ and that failure in mode 2 does not occur before $n T$, as illustrated in Fig. 2. The corresponding occurrence probability of this scenario is

$$
\begin{aligned}
P\left(T_{c}=n T\right) & =\operatorname{Pr}\left\{\begin{array}{l}
\left\{(n-1) T<X_{1}<n T, X_{1}+X_{2}>n T\right\} \\
\cup\left\{X_{1}>n T\right\}
\end{array}\right\} \operatorname{Pr}\left\{X_{3}>n T\right\} \\
& =R_{3}(n T)\left(\int_{(n-1) T}^{n T} f_{1}(t) R_{2}(n T-t) d t+R_{1}(n T)\right) .
\end{aligned}
$$
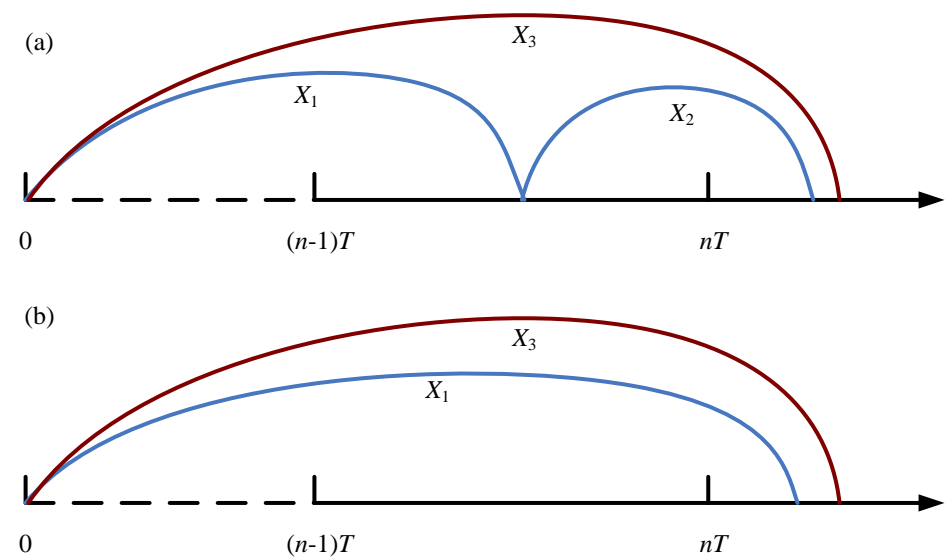

Fig.2 Renewal of the system due to the preventive replacement: (a) defective state of failure mode 1 before $n T$; (b) normal state of failure mode 1 before $n T$.

As previously mentioned, whenever a failure occurs, the system is renewed immediately. A failure occurring at $t \in((k-1) T, k T)$ implies the following two exclusive cases: 
(i) The system due to failure mode 1 enters the defective state at some $\tau \in((k-1) T, t)$ and leads to the system failure at $t$, while failure mode 2 does not occur before $t$, as illustrated in Fig. 3(a);

(ii) Failure mode 2 leads to the system failure at $t$, and the system due to failure mode 1 is either in the normal state or the defective state at $t$ (but is normal at $(k-1) T)$, as illustrated in Fig. 3(b) and Fig. 3(c).

Let $T_{f}$ be the time of system failure. The PDF of $T_{f}$ is given as

$$
\begin{aligned}
& f_{T_{f}}^{k}(t)= \lim _{\Delta t \rightarrow 0} \frac{1}{\Delta t} \operatorname{Pr}\left\{\begin{array}{l}
\left\{(k-1) T<X_{1}<k T, X_{1}+X_{2} \in(t, t+\Delta t), X_{3}>t\right\} \\
\cup\left\{(k-1) T<X_{1}, X_{1}+X_{2}>t, X_{3} \in(t, t+\Delta t)\right\}
\end{array}\right\} \\
&=R_{3}(t) \int_{(k-1) T}^{t} f_{1}(\tau) f_{2}(t-\tau) d \tau \\
& \quad+f_{3}(t)\left(R_{1}((k-1) T)-\int_{(k-1) T}^{k T} f_{1}(\tau) F_{2}(t-\tau) d \tau\right), \\
& t \in((k-1) T, k T), k=1, \ldots, n .
\end{aligned}
$$

The probability that the system fails in $((k-1) T, k T)$ is

$$
\begin{aligned}
& \operatorname{Pr}\left\{(k-1) T<T_{f}<k T\right\}=\int_{(k-1) T}^{k T} f_{T_{f}}^{k}(t) d t \\
& =\int_{(k-1) T}^{k T} \int_{(k-1) T}^{t} f_{1}(\tau)\left(R_{3}(t) f_{2}(t-\tau)-f_{3}(t) F_{2}(t-\tau)\right) d \tau d t \\
& \quad+R_{1}((k-1) T)\left(F_{3}(k T)-F_{3}((k-1) T)\right) \\
& =R_{3}(k T) \int_{(k-1) T}^{k T} f_{1}(\tau) F_{2}(k T-\tau) d \tau \\
& \quad+R_{1}((k-1) T)\left(F_{3}(k T)-F_{3}((k-1) T)\right) \\
& =R_{1}((k-1) T) R_{3}((k-1) T)-R_{1}(k T) R_{3}(k T) \\
& \quad-R_{3}(k T) \int_{(k-1) T}^{k T} f_{1}(\tau) R_{2}(k T-\tau) d \tau .
\end{aligned}
$$



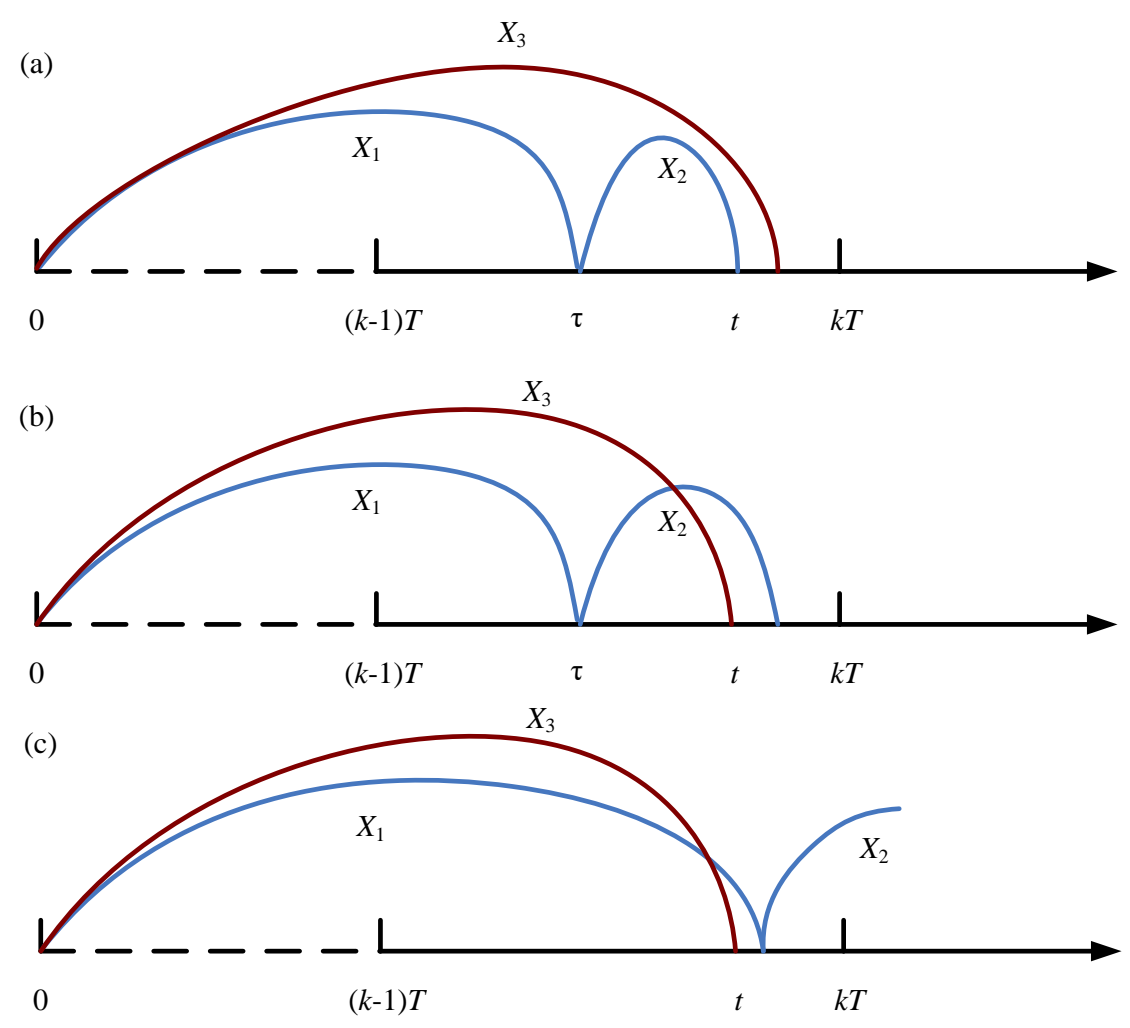

Fig.3 Renewal of the system due to a failure of the system. (a) Failure is due to failure mode 1. (b) and (c): Failure is due to failure mode 2.

\subsection{Expected length and cost of a renewal cycle}

With the above analysis, the expected length of a renewal cycle, $T_{c}$, can be obtained as

$$
\begin{aligned}
E\left(T_{c}\right) & =\sum_{k=1}^{n} k T P\left(T_{c}=k T\right)+\sum_{k=1}^{n} \int_{(k-1) T}^{k T} t f_{T_{f}}^{k}(t) d t \\
& =\int_{0}^{n T} R_{1}(t) R_{3}(t) d t+\sum_{k=1}^{n} \int_{(k-1) T}^{k T} R_{3}(t) \int_{(k-1) T}^{t} f_{1}(\tau) R_{2}(t-\tau) d \tau d t .
\end{aligned}
$$

The term $\sum_{k=1}^{n-1} k T P\left(T_{c}=k T\right)$ corresponds to the contribution of the detected defective state of failure mode $1, n T P\left(T_{c}=n T\right)$ denotes the contribution from the preventive replacement and $\sum_{k=1}^{n} \int_{(k-1) T}^{k T} t f_{T_{f}}^{k}(t) d t$ represents the contribution due to the failure renewal. 
With the corresponding probabilities derived in Section 3.1, the expected cost in a renewal cycle can be readily obtained as

$$
\begin{aligned}
& E\left(C_{c}\right)=\sum_{k=1}^{n-1}\left(k C_{I}+C_{R}\right) P\left(T_{c}=k T\right)+\left((n-1) C_{I}+C_{R}\right) P\left(T_{c}=n T\right) \\
& \quad+\sum_{k=1}^{n}\left((k-1) C_{I}+C_{F}\right) \operatorname{Pr}\left\{(k-1) T<T_{f}<k T\right\} \\
& =C_{I} \sum_{k=1}^{n-1} R_{3}(k T)\left(R_{1}(k T)+\int_{(k-1) T}^{k T} f_{1}(t) R_{2}(k T-t) d t\right) \\
& +C_{R}\left(R_{3}(n T) R_{1}(n T)+\sum_{k=1}^{n} R_{3}(k T) \int_{(k-1) T}^{k T} f_{1}(t) R_{2}(k T-t) d t\right) \\
& +C_{F}\left(1-R_{3}(n T) R_{1}(n T)-\sum_{k=1}^{n} R_{3}(k T) \int_{(k-1) T}^{k T} f_{1}(t) R_{2}(k T-t) d t\right) .
\end{aligned}
$$

Here, $\sum_{k=1}^{n-1}\left(k C_{I}+C_{R}\right) P\left(T_{c}=k T\right)$ represents the expected cost attributable to the inspection (the cost of $k$ inspections) and a replacement when the defective state of failure mode 1 is detected, $\left((n-1) C_{I}+C_{R}\right) P\left(T_{c}=n T\right)$ corresponds to the expected cost of the preventive replacement together with the expected cost of $(n-1)$ inspections before it and $\sum_{k=1}^{n}\left((k-1) C_{I}+C_{F}\right) P\left\{(k-1) T<T_{f}<k T\right\}$ corresponds to the scenario that the system fails, including the expected cost of the inspection before failure and the expected cost caused by failure.

\subsection{Optimal solution}

Let $g(T, n)=E\left(T_{c}\right)$ and $h(T, n)=E\left(C_{c}\right)$. We can have the following properties in terms of $g(T, n)$ and $h(T, n)$.

Proposition 1. $g(T, n)$ is monotonically increasing and bounded with respect to $n$. In addition,

$$
g(T, n)<\int_{0}^{n T} R_{3}(t) d t
$$

Detailed proof is shown in Appendix A. With Proposition 1, we can easily have 
$\lim _{n \rightarrow \infty} g(T, n)<E\left(T_{3}\right)$. Therefore, when the preventive replacement is postponed, the expected cycle length will always increase; however, the maximum will not exceed the expectation of $X_{3}$, i.e., it is bottlenecked by failure mode 2 .

Proposition 2. $h(T, n)$ is monotonically increasing and bounded with respect to $n$.

$$
h(T, n)<\frac{C_{I}}{T} \int_{0}^{(n-1) T} R_{3}(t) d t+C_{F}
$$

Detailed proof is shown in Appendix B. Proposition 2 implies that when the preventive replacement is postponed, the possibility that the system is renewed by a failure is increased, which in turn increases the cost resulting from failures. On the contrary, the possibility that the system is renewed by a replacement is decreased, and the expected cost of replacements is decreased. Nevertheless, the postponed preventive replacement always increases the expected inspection cost, since it extends the expected length of the renewal cycle. We can also have

$$
\lim _{n \rightarrow \infty} h(T, n)<\frac{C_{I} E\left(X_{3}\right)}{T}+C_{F}
$$

As for the inspection period $T$, the corresponding derivatives of $g(T, n)$ and $h(T, n)$ can be obtained after tedious derivations. For $g(T, n)$, we have

$$
\begin{aligned}
& \Delta_{T} g(T, n)=n R_{1}(n T) R_{3}(n T) \\
& +\sum_{k=1}^{n}\left(\begin{array}{l}
k R_{3}(k T) \int_{(k-1) T}^{k T} f_{1}(t) R_{2}(k T-t) d t \\
-(k-1) f_{1}((k-1) T) \int_{(k-1) T}^{k T} R_{3}(t) R_{2}(t-(k-1) T) d t
\end{array}\right)
\end{aligned}
$$

For $h(T, n)$, we have 


$$
\begin{aligned}
& \Delta_{T} h(T, n)=-C_{I}\left(A_{n}+\sum_{k=1}^{n-1} k f_{3}(k T) R_{1}(k T)\right) \\
& +\left(C_{F}-C_{R}\right)\left(A_{n}-\sum_{k=1}^{n-1} k f_{1}(k T) R_{3}(k T)+n f_{3}(n T) R_{1}(n T)\right)
\end{aligned}
$$

where

$$
A_{n}=\sum_{k=1}^{n}\left(\begin{array}{l}
k f_{3}(k T) \int_{(k-1) T}^{k T} f_{1}(t) R_{2}(k T-t) d t+k R_{3}(k T) \int_{(k-1) T}^{k T} f_{1}(t) f_{2}(k T-t) d t \\
-(k-1) f_{1}((k-1) T) R_{2}(T) R_{3}(k T)
\end{array}\right)
$$

Then, based on $\Delta_{n} g(T, n), \Delta_{n} h(T, n), \Delta_{T} g(T, n)$ and $\Delta_{T} h(T, n)$, the optimal inspection and replacement strategy can be readily found. Let $f(T, n)=E C R(T, n)=h(T, n) / g(T, n)$, we can have

$$
\begin{gathered}
\Delta_{n} f(T, n)=f(T, n+1)-f(T, n)=\frac{h(T, n+1)}{g(T, n+1)}-\frac{h(T, n)}{g(T, n)} \\
\Delta_{T} f(T, n)=\frac{\Delta_{T} h(T, n) g(T, n)-\Delta_{T} g(T, n) h(T, n)}{(g(T, n))^{2}}
\end{gathered}
$$

Based on $\Delta_{n} f(T, n)$ and $\Delta_{T} f(T, n)$, the optimal $(T, n)$ that minimizes $\operatorname{ECR}(T, n)$ can be obtained straightforward. As $n$ is discrete, we can first find an optimal $T_{n}^{*}$ that minimizes $f(T, n)$ for fixed $n$, and then find the optimal $n^{*}$ that minimizes $f\left(T_{n}^{*}, n\right)$. Carrying on this procedure iteratively, we can find the optimal $\left(T^{*}, n^{*}\right)$

\section{Maintenance model with imperfect inspections}

In this section, we consider the effect of imperfect inspections $(\beta<1)$. Denote $N_{d}$ as the number of inspections taken to detect the defective state after the occurrence of a defective state. Obviously, $N_{d}$ follows the geometric distribution 
$P\left(N_{d}=i\right)=(1-\beta)^{i-1} \beta$ (Williams \& Hirani, 1997). Similar as in Section 3, we consider the following three exhaustive renewal scenarios: (1) the renewal results from the detection of the defective state; (2) the renewal results from the preventive replacement at the $n$th inspection; and (3) the renewal cycle results from a failure.

(1) Consider the first scenario (where a defective state is discovered at the $k$ th inspection, $k=1,2, \ldots, n-1)$. Here, the system does not fail, but rather falls into the defective state before $k T$. In addition, we have $N_{d}=k-\left\lfloor X_{1} / T\right\rfloor$, where $\lfloor x\rfloor$ gives the maximum integer not bigger than $x$. The occurrence probability of this scenario can be obtained as

$$
\begin{aligned}
P\left(T_{c}=k T\right) & =\operatorname{Pr}\left\{X_{3}>k T\right\} \cdot \operatorname{Pr}\left\{X_{1}+X_{2}>k T, X_{1}<k T, N_{d}=k-\left\lfloor X_{1} / T\right\rfloor\right\} \\
& =R_{3}(k T) \int_{0}^{k T} f_{1}(t) R_{2}(k T-t)(1-\beta)^{k-t / T\rfloor-1} \beta d t \\
& =R_{3}(k T) \sum_{i=1}^{k} \int_{(i-1) T}^{i T} f_{1}(t) R_{2}(k T-t)(1-\beta)^{k-i} \beta d t .
\end{aligned}
$$

(2) Consider the second scenario (where the system is replaced at the $n$th inspection if no failure occurs and no defective state is detected before $n T$ ). The event that no defective state is detected consists of two scenarios: the system is in the normal state, or the system is in the defective state but has not been discovered. Clearly, we have $N_{d} \geq n-\left\lfloor X_{1} / T\right\rfloor$, denoting that no defective state is detected before $n T$ given that the system is in the defective state. The probability of this event can be obtained as

$$
\begin{aligned}
& P\left(T_{c}=n T\right)=\operatorname{Pr}\left\{X_{3}>n T\right\} \\
& \quad \cdot \operatorname{Pr}\left\{\left(X_{1}+X_{2}>n T, X_{1}<n T, N_{d} \geq n-\left\lfloor X_{1} / T\right\rfloor\right) \cup\left(X_{1}>n T\right)\right\} \\
& =R_{3}(n T)\left(\int_{0}^{n T} f_{1}(t) R_{2}(n T-t)(1-\beta)^{n-\lfloor t T\rfloor-1} d t+R_{1}(n T)\right) \\
& =R_{3}(n T)\left(\sum_{i=1}^{n} \int_{(i-1) T}^{i T} f_{1}(t) R_{2}(n T-t)(1-\beta)^{n-i} d t+R_{1}(n T)\right) .
\end{aligned}
$$

(3) Consider the third scenario (where either failure mode 1 or failure mode 2 leads to system failure). The PDF that a failure occurs by time $t,(k-1) T<t<k T$ is expressed as 


$$
\left.\begin{array}{rl}
f_{T_{f}}^{k}(t) & =\lim _{\Delta t \rightarrow 0} \frac{1}{\Delta t} \operatorname{Pr}\left\{\begin{array}{l}
\left(X_{1}+X_{2} \in(t, t+\Delta t), X_{1} \leq(k-1) T, X_{3}>t, N_{d}>k-1-\left\lfloor X_{1} / T\right\rfloor\right) \\
\cup\left(X_{1}+X_{2} \in(t, t+\Delta t), t>X_{1}>(k-1) T, X_{3}>t\right) \\
\cup\left(X_{1}+X_{2}>t, X_{1} \leq(k-1) T, N_{d}>k-1-\left\lfloor X_{1} / T\right\rfloor, X_{3} \in(t, t+\Delta t)\right) \\
\cup\left(X_{1}+X_{2}>t, X_{1}>(k-1) T, X_{3} \in(t, t+\Delta t)\right)
\end{array}\right.
\end{array}\right\}
$$

If $\beta$ is set to 1 for perfect inspection, the failure probability density of Eq. (11) is identical to that of Eq. (5).

The renewal is resulted from either preventive replacement or corrective replacement due to unexpected failures. After some simplifications, the expected length of a renewal cycle is expressed as

$$
\begin{aligned}
& E\left(T_{c}\right)=\sum_{k=1}^{n} k T P\left(T_{c}=k T\right)+\sum_{k=1}^{n} \int_{(k-1) T}^{k T} t f_{T_{f}}^{k}(t) d t \\
& =\sum_{k=1}^{n-1} k T R_{3}(k T) \sum_{i=1}^{k} \int_{(i-1) T}^{i T} f_{1}(t) R_{2}(k T-t)(1-\beta)^{k-i} \beta d t \\
& +n T R_{3}(n T) \sum_{j=1}^{n} \int_{(j-1) T}^{j T} f_{1}(\tau) R_{2}(n T-\tau)(1-\beta)^{n-j} d \tau \\
& -\sum_{k=1}^{n} \int_{(k-1) T}^{k T} t R_{3}(t) \sum_{j=1}^{k-1} \int_{(j-1) T}^{j T} f_{1}(\tau) R_{2}(t-\tau)(1-\beta)^{k-j} d \tau d t \\
& +\sum_{k=1}^{n} k T R_{3}(k T) \int_{(k-1) T}^{k T} f_{1}(\tau) F_{2}(k T-\tau) d \tau \\
& \quad-\sum_{k=1}^{n} \int_{(k-1) T}^{k T} \int_{(k-1) T}^{t} f_{1}(\tau) F_{2}(t-\tau) R_{3}(t) d \tau d t \\
& +\sum_{k=1}^{n} R_{1}((k-1) T) \int_{(k-1) T}^{k T} t f_{3}(t) d t+n T R_{3}(n T) R_{1}(n T) .
\end{aligned}
$$

The expected cost in a renewal cycle can be obtained as 


$$
\begin{aligned}
& E\left(C_{c}\right)=\sum_{k=1}^{n}\left(k C_{I}+C_{R}\right) R_{3}(k T) \sum_{i=1}^{k} \int_{(i-1) T}^{i T} f_{1}(t) R_{2}(k T-t)(1-\beta)^{k-i} \beta d t \\
& -C_{I} R_{3}(n T)\left(\sum_{i=1}^{n} \int_{(i-1) T}^{i T} f_{1}(t) R_{2}(n T-t)(1-\beta)^{n-i} d t\right)+\left((n-1) C_{I}+C_{R}\right) R_{3}(n T) R_{1}(n T) \\
& +\sum_{i=1}^{n}\left((i-1) C_{I}+C_{F}\right) \int_{(i-1) T}^{i T} R_{3}(t) \sum_{j=1}^{k-1} \int_{(j-1) T}^{j T} f_{1}(\tau) f_{2}(t-\tau)(1-\beta)^{k-j} d \tau d t \\
& +\sum_{i=1}^{n}\left((i-1) C_{I}+C_{F}\right) \int_{(i-1) T}^{i T} f_{3}(t) \sum_{j=1}^{k-1} \int_{(j-1) T}^{j T} f_{1}(\tau) R_{2}(t-\tau)(1-\beta)^{k-j} d \tau d t \\
& +\sum_{i=1}^{n}\left((i-1) C_{I}+C_{F}\right) \int_{(i-1) T}^{i T} R_{3}(t) \int_{(k-1) T}^{t} f_{1}(\tau) f_{2}(t-\tau) d \tau d t \\
& +\sum_{i=1}^{n}\left((i-1) C_{I}+C_{F}\right) \int_{(i-1) T}^{i T} f_{3}(t)\left(R_{1}((k-1) T)-\int_{(k-1) T}^{t} f_{1}(\tau) F_{2}(t-\tau) d \tau\right) \tau d t
\end{aligned}
$$

With the expression of $E\left(T_{c}\right)$ and $E\left(C_{c}\right)$, we can derive the ELRCR according to Eq. (2). Then, with the given $T$ and $n$, one can easily calculate the corresponding long-run cost rate. The optimal $\left(T^{*}, n^{*}\right)$ that minimizes the ELRCR can be easily derived with numerical methods. The current model can be extended to the cases with arbitrary preventive replacement interval and with time-dependent inspection probability. We present these extensions in Appendix C and Appendix D for compactness of the paper.

\section{Case Study}

Infusion pumps are important equipment to pump fluids for patients. Infusion pumps contain a variety of types, among which the widely used type is the peristaltic pump. A peristaltic pump usually suffers two failure modes. One is due to the battery which is routinely checked up of its voltage, the other is the electrical parts failure which cannot be monitored. The battery goes through a degradation process before failure, which can be described with a delay-time failure model, while the electrical parts are subject to sudden failures (Wang, 1992). Under the proposed framework, we use $X_{1}$ 
and $X_{2}$ to denote the duration of the normal state and the deterioration state of the battery and $X_{3}$ to denote the lifetime of the electrical parts. The three variables are assumed to follow Weibull distributions $F_{i}(x)=1-\exp \left\{-\left(x / \eta_{i}\right)^{\beta_{i}}\right\}$, where the distribution parameters are as given in Table 1. The inspection cost, replacement cost and failure cost are set as $C_{I}=10, C_{R}=100$ and $C_{F}=800$, respectively.

Table 1 Distribution parameters for lifetime distributions of the peristaltic infusion pump.

\begin{tabular}{cccc}
\hline$i$ & 1 & 2 & 3 \\
\hline$\beta_{i}$ & 1.5 & 1.2 & 2 \\
\hline$\eta_{i}$ & 2 & 1 & 2.5 \\
\hline
\end{tabular}

\subsection{Illustration of the model proposed in Section 3}

With the given parameter setting, the optimal inspection interval and preventive replacement interval are obtained as $\left(T^{*}, n^{*}\right)=(0.23,6)$. This indicates that the optimal inspection interval is 0.23 and that preventive replacement should be carried out at the sixth inspection if no failure occurs before it. The expected cycle length and the expected cycle cost are $E\left(T_{c}\right)=0.6165$ and $E\left(C_{c}\right)=98.28$, respectively, while the optimal ELRCR is $\operatorname{ECR}\left(T^{*}, n^{*}\right)=152.2$. Fig. 4 shows how the ELRCR varies in terms of $n$ and $T$. 

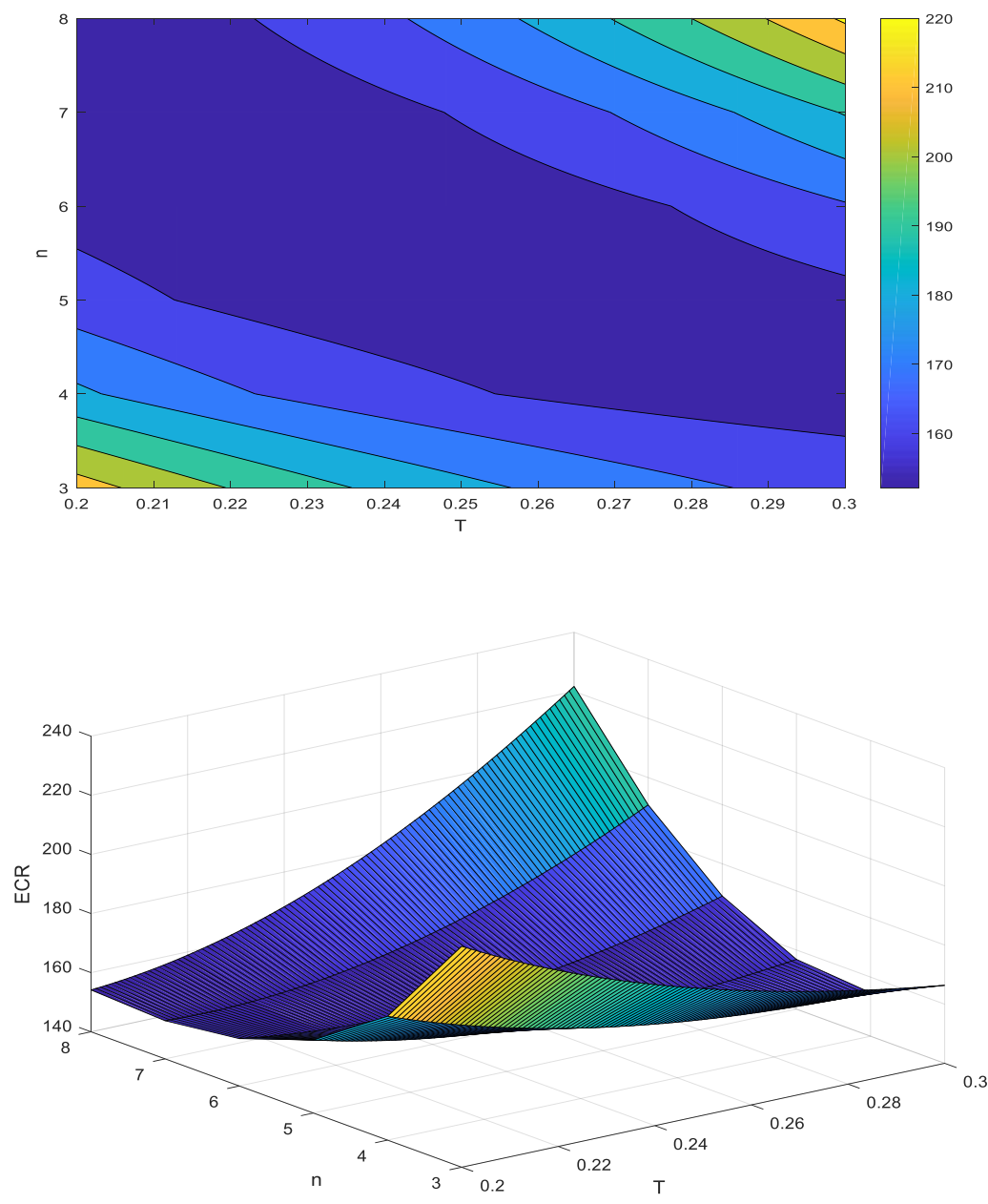

Fig.4 Variation of ELRCR in terms of $n$ and $T$

To show the influence of the cost parameters on the optimal inspection and maintenance strategy, Fig. gives the variation of the optimal $\left(T^{*}, n^{*}\right)$ and $\operatorname{ECR}\left(T^{*}, n^{*}\right)$ for different inspection cost $C_{I}$ and replacement cost $C_{R}$. It is shown that the optimal inspection interval $T^{*}$ increases monotonically with $C_{I}$, indicating that the inspection tends to be less frequent as the unit inspection cost increases. In addition, the optimal inspection interval $T^{*}$ decreases with the replacement cost $C_{R}$. Actually, the cost for inspection is relatively cheaper when the cost for replacement increases, thus it justifies a smaller inspection interval. More frequent inspections (i.e., a smaller 
inspection interval) can attenuate the risk of system failure and thus reduce the maintenance cost, which is more effective for the system with a higher replacement cost. In contrast, as the inspection becomes less frequent, $n^{*}$ decreases to ensure that the risk of failure due to failure mode 1 can be controlled under a certain level. With an increased inspection interval, the number of inspections should be decreased so as to balance the probability of failure. This logic is illustrated in the middle panel of Fig., where the optimal preventive replacement interval $n^{*}$ decreases with the inspection cost $C_{I}$ and decreases with the replacement cost $C_{R}$. Clearly, ELRCR always increases with $C_{I}$ and $C_{R}$.
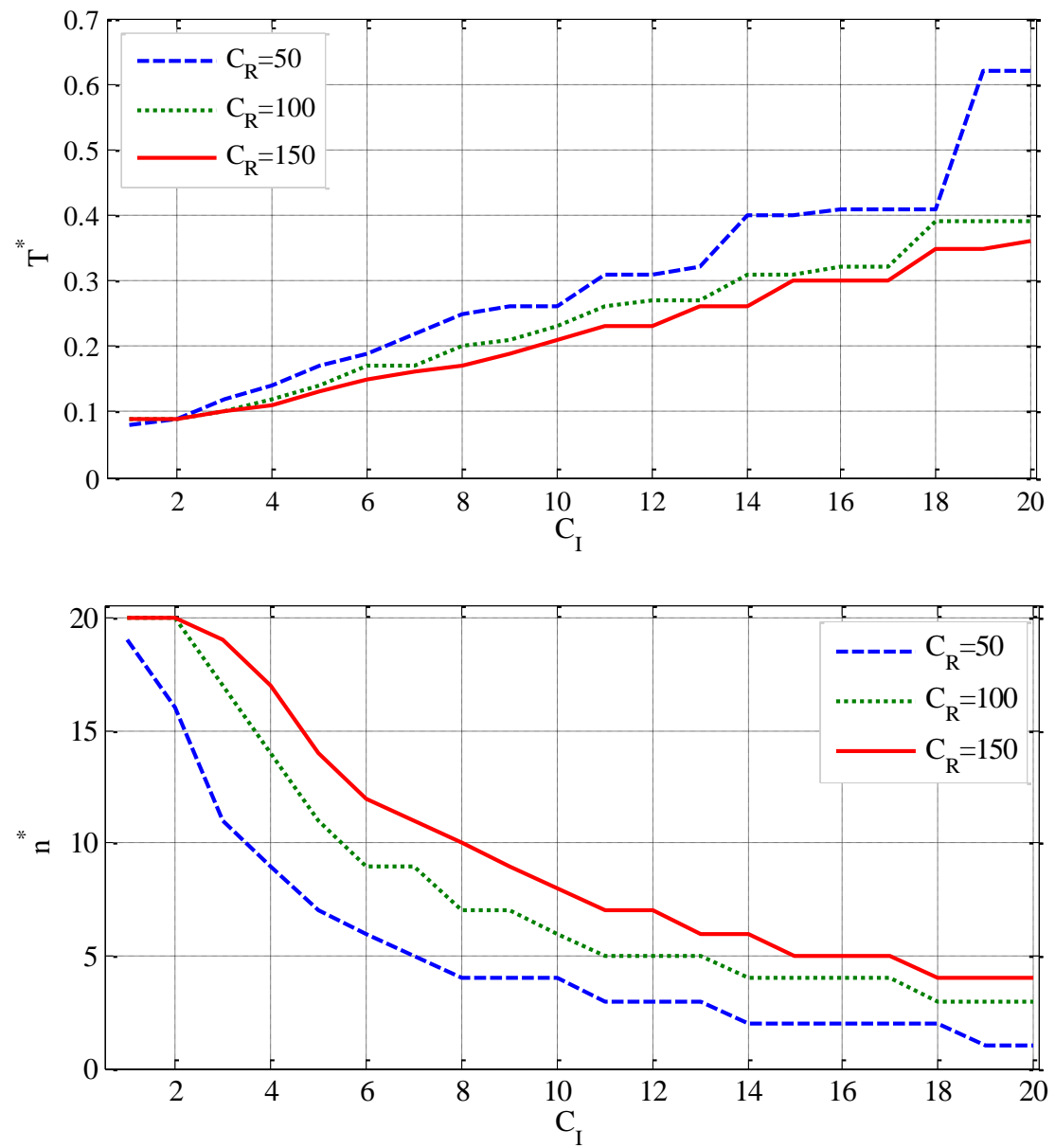


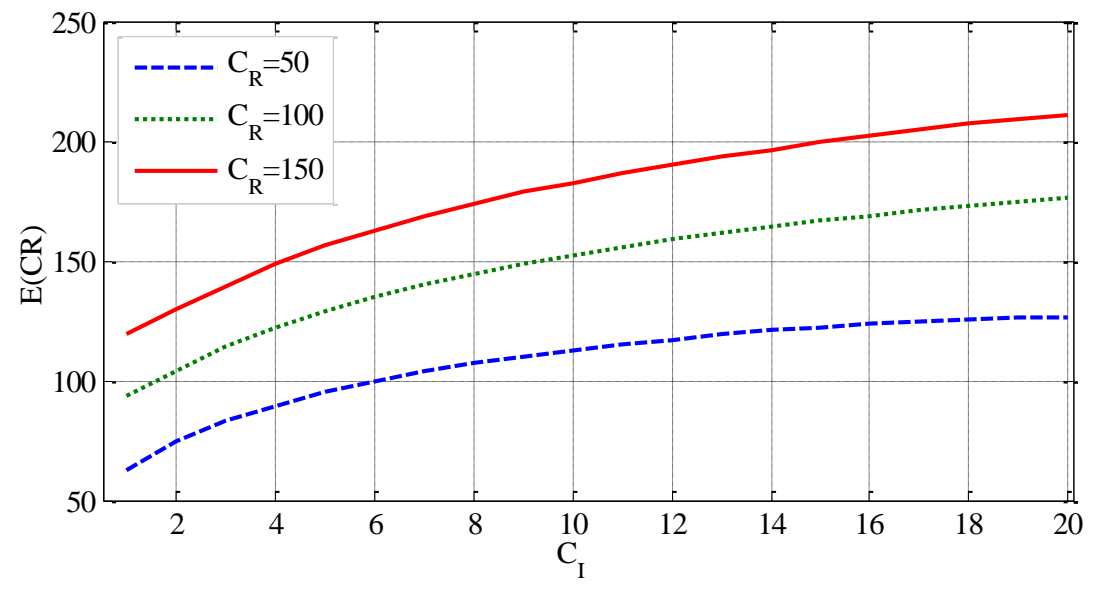

Fig.5 Variation of the optimal $\left(T^{*}, n^{*}\right)$ and the optimal ELRCR with respect to the inspection cost $C_{I}$ for different $C_{R}$.

\subsection{Illustration of the model proposed in Section 4}

Consider the case with imperfect inspections. The probability of detection is set as $\beta=0.7$. In this setting, the optimal inspection interval $T^{*}$ and the optimal preventive replacement cycle $n^{*}$ are obtained as $\left(T^{*}, n^{*}\right)=(0.27,5)$. The associated expected cost in a renewal cycle $E\left(C_{c}\right)$ and length of a cycle length $E\left(T_{c}\right)$ are obtained as $E\left(C_{c}\right)=118.7$ and $E\left(T_{c}\right)=0.7431$, respectively. The optimal ELRCR is achieved as $\operatorname{ECR}\left(T^{*}, n^{*}\right)=159.8$. Fig.6 presents how the ELRCR varies with different $n$ and $T$.

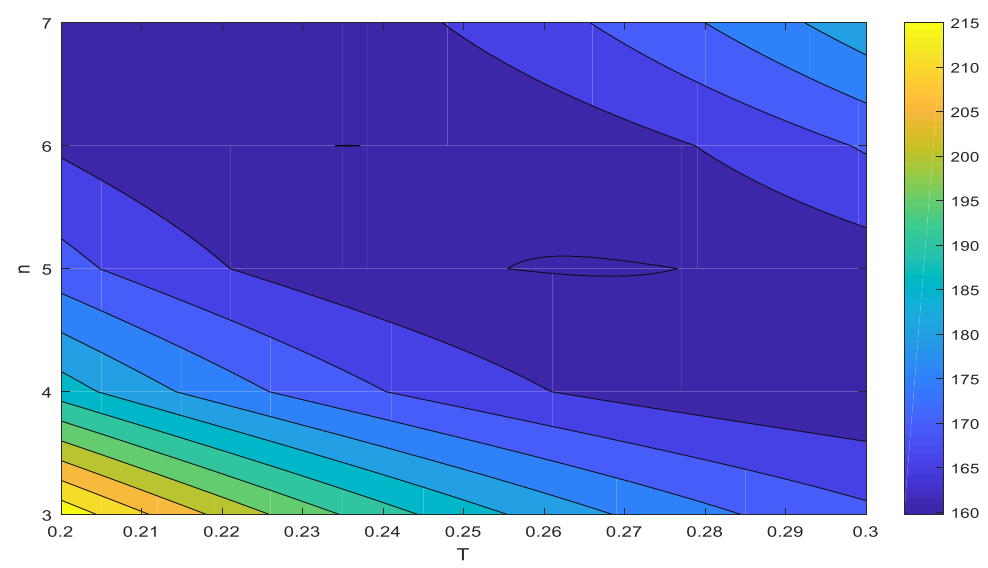




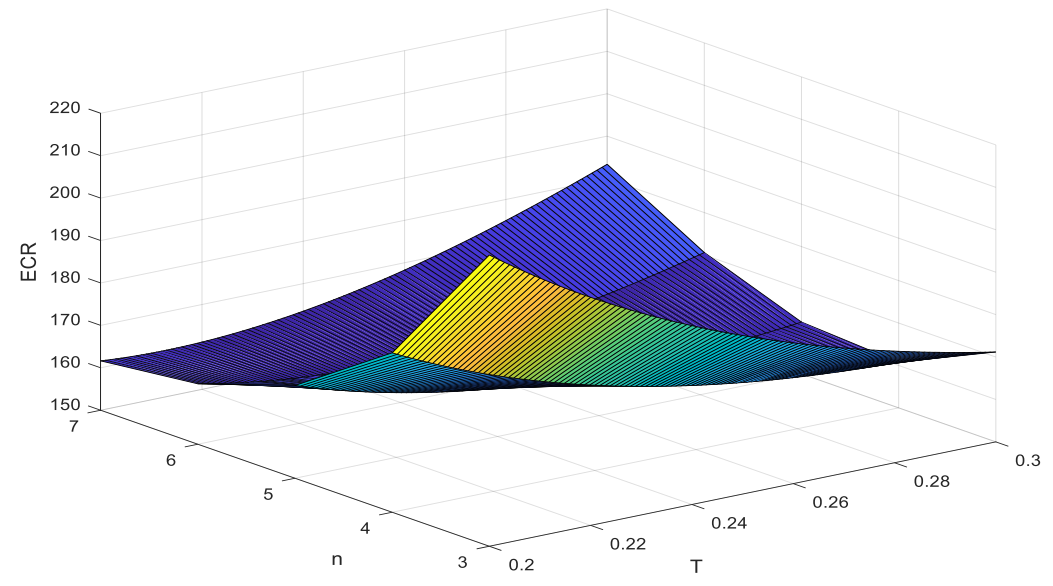

Fig.6 Variation of ELRCR with respect to $n$ and $T$

Compared with the scenario of perfect inspection in Section 5.1, the existence of imperfect inspection leads to a larger ELRCR. This is because the failed detection of the defective state increases both the renewal cycle length and the maintenance cost in a renewal cycle. In contrast, the preventive replacement interval $n^{*}$ is smaller, as the imperfect inspection increases the risk of failure; thus, the system should be preventively replaced more frequently. In addition, we plot the variations of $\left(T^{*}, n^{*}\right)$ and the corresponding $\operatorname{ECR}\left(T^{*}, n^{*}\right)$ with respect to different inspection cost $C_{I}$ and replacement cost $C_{R}$, as shown in Fig.. It is obvious that $\operatorname{ECR}\left(T^{*}, n^{*}\right)$ increases with the inspection cost $C_{I}$ and replacement cost $C_{R}$. However, the monotonic trend of the optimal preventive replacement interval $n^{*}$ and optimal inspection interval $T^{*}$ is not as apparent as that for the basic model of Section 3. It can be seen that $n^{*}$ decreases with $C_{I}$ and $T^{*}$ increases with $C_{I}$. Yet, the trend with respect to $C_{R}$ is somewhat obscure, especially for the case where the inspection cost $C_{I}$ is small. 

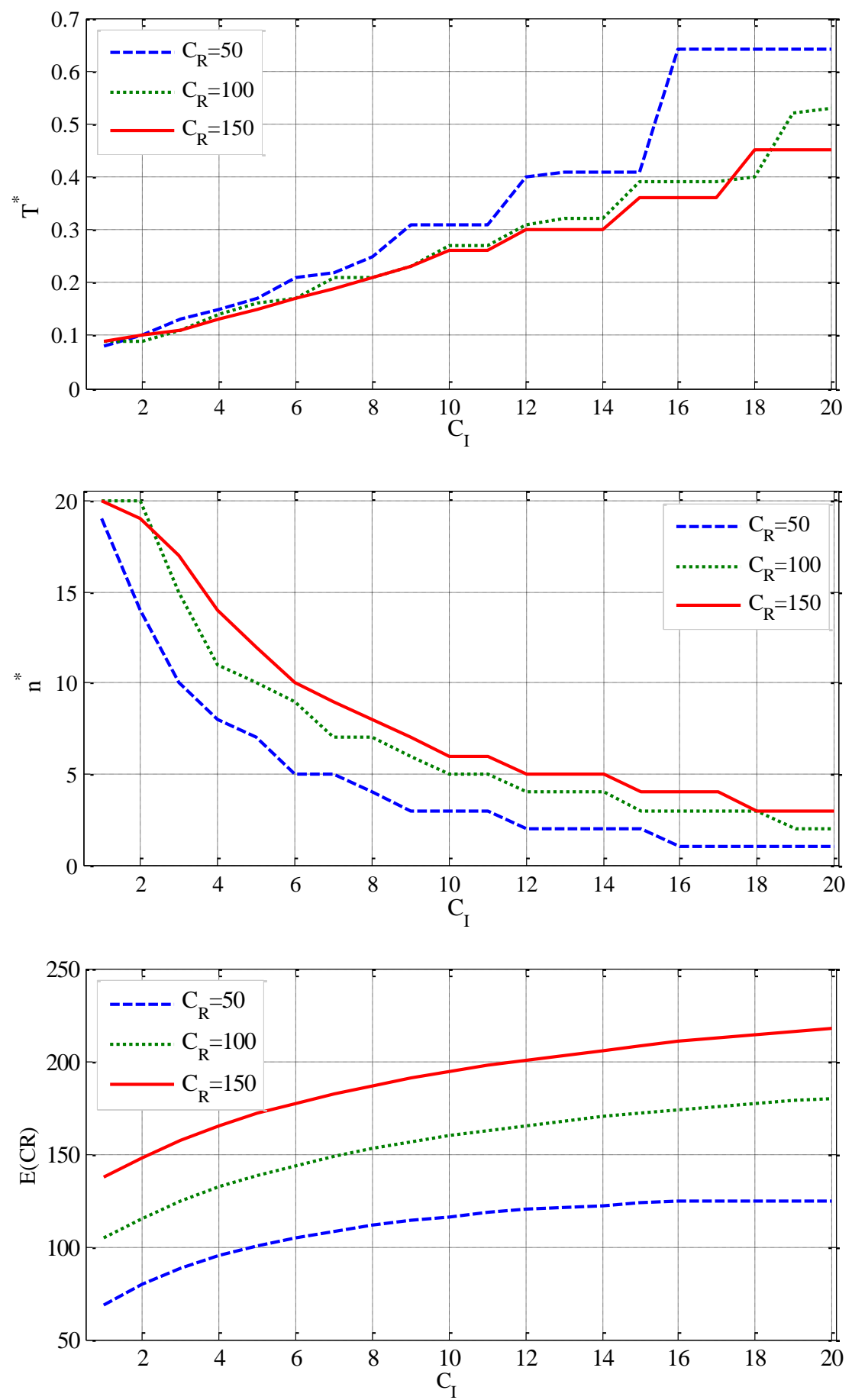

Fig.7 Variation of the optimal $\left(T^{*}, n^{*}\right)$ and the optimal ELRCR with respect to $C_{I}$ and $C_{R}$ for imperfect inspection.

To investigate the effect of $\beta$ on the optimal maintenance policy, we plot the 
variation of the optimal $\left(T^{*}, n^{*}\right)$ and the corresponding $\operatorname{ECR}\left(T^{*}, n^{*}\right)$ with the detection probability $\beta$, as shown in Fig.. From Fig., we can see that $\operatorname{ECR}\left(T^{*}, n^{*}\right)$ decreases monotonically with respect to $\beta$, which indicates that an improved detection accuracy contributes to the reduction of maintenance cost. The optimal inspection $T^{*}$ shows a non-increasing trend with $\beta$, while the optimal preventive replacement cycle $n^{*}$ shows a non-decreasing trend with $\beta$. This is because, when the detection probability is small, inspection should be carried out less frequently as the effect of inspection is not significant. Instead, more effort should be placed on the preventive replacement, and a more frequent preventive replacement is advocated. The sensitivity analysis on $\beta$ implies that companies should pay more effort into improving the detection accuracy, so as to reduce the maintenance cost.

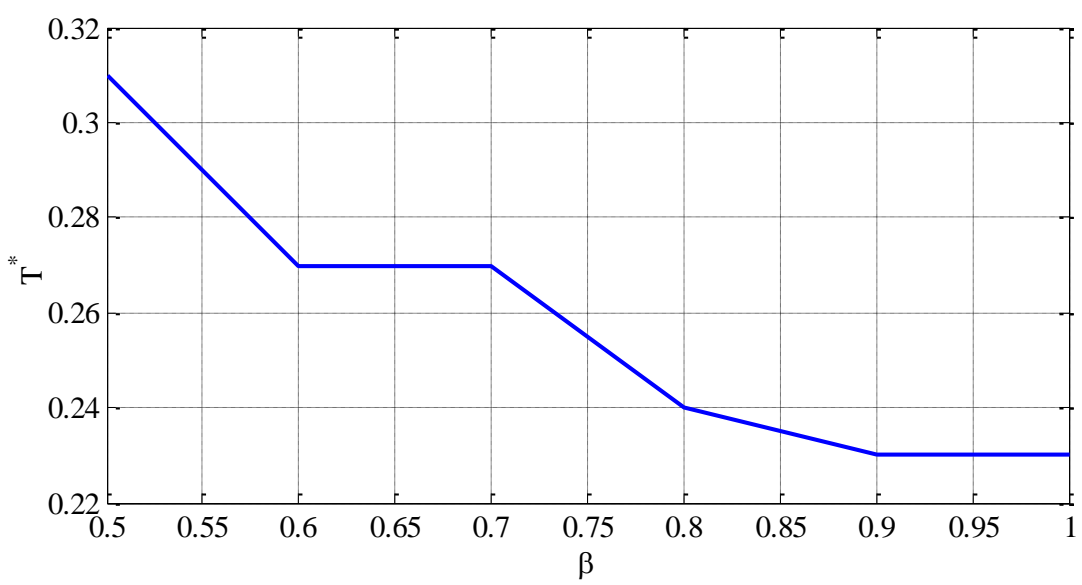



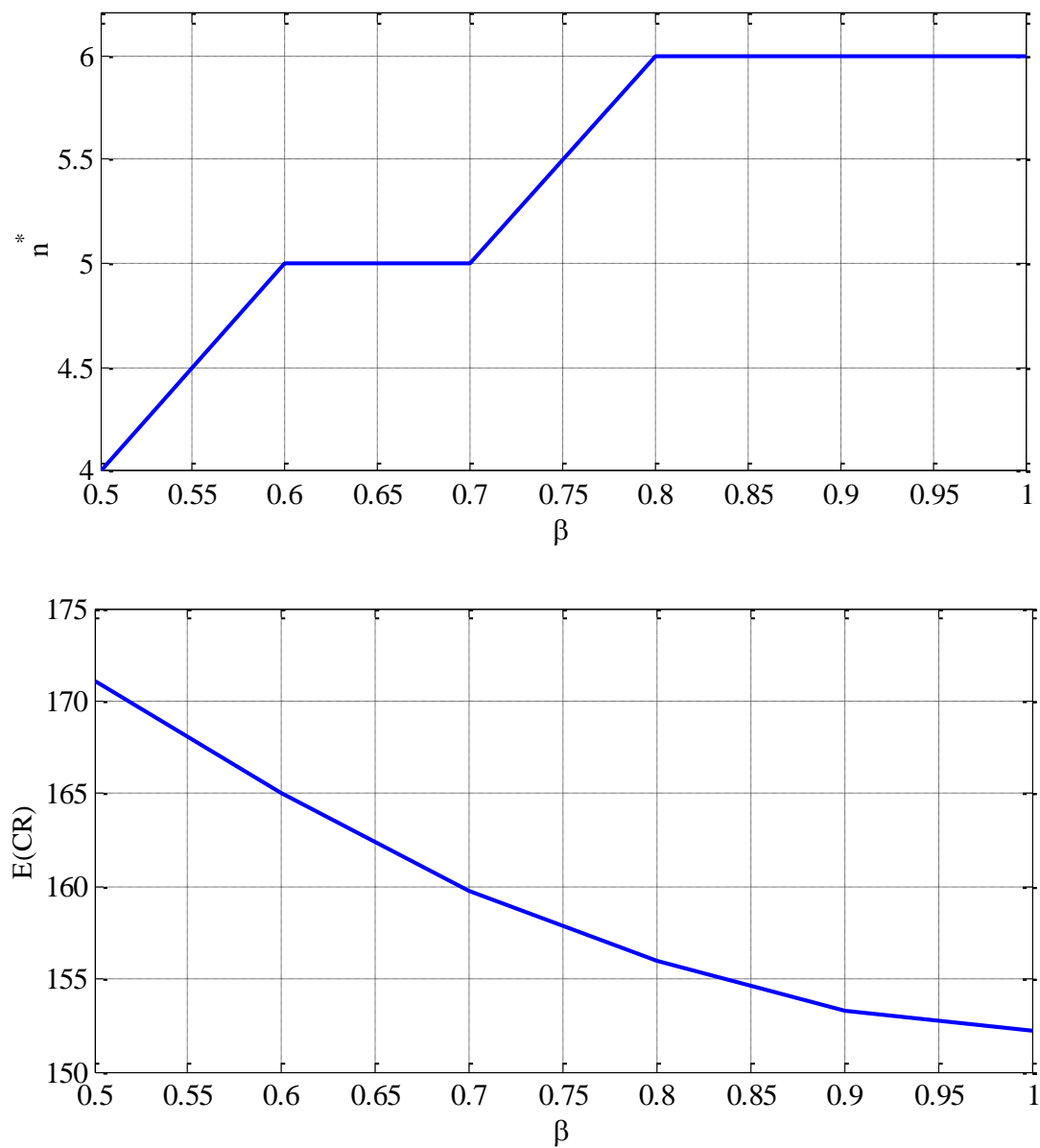

Fig. 8 Variation of the optimal $\left(T^{*}, n^{*}\right)$ and the optimal ELRCR with respect to the detection probability $\beta$.

\subsection{Comparison with block replacement and age-based maintenance}

To show the effectiveness of the proposed maintenance policy, we compare the proposed maintenance policy with two traditional maintenance policies: block replacement and age-based maintenance policy. Block replacement policy implies that the system is replaced at failure, while no preventive replacement and inspection is implemented to prevent unexpected failures. With the block replacement policy, the expected length of a renewal cycle is 1.727 and the ELRCR is obtained as $E C R=463.22$.

Age-based maintenance indicates that the system is replaced either at failure or at 
a specific age. Fig. 7 shows how the ELRCR varies with different replacement age. It can be observed that the optimal age-based maintenance policy is achieved when the replacement age is 0.73 . The expected length of a renewal cycle and the expected cost in a renewal cycle are given as 0.7014 and 183.94, respectively. The optimal ELRCR is obtained as $E C R^{*}=262.23$. Compared with these two maintenance policies, it can be concluded that the proposed maintenance policy is more effective in reducing the maintenance cost. In addition, the results imply the importance of inspection for a system with the delay-time failure mode.

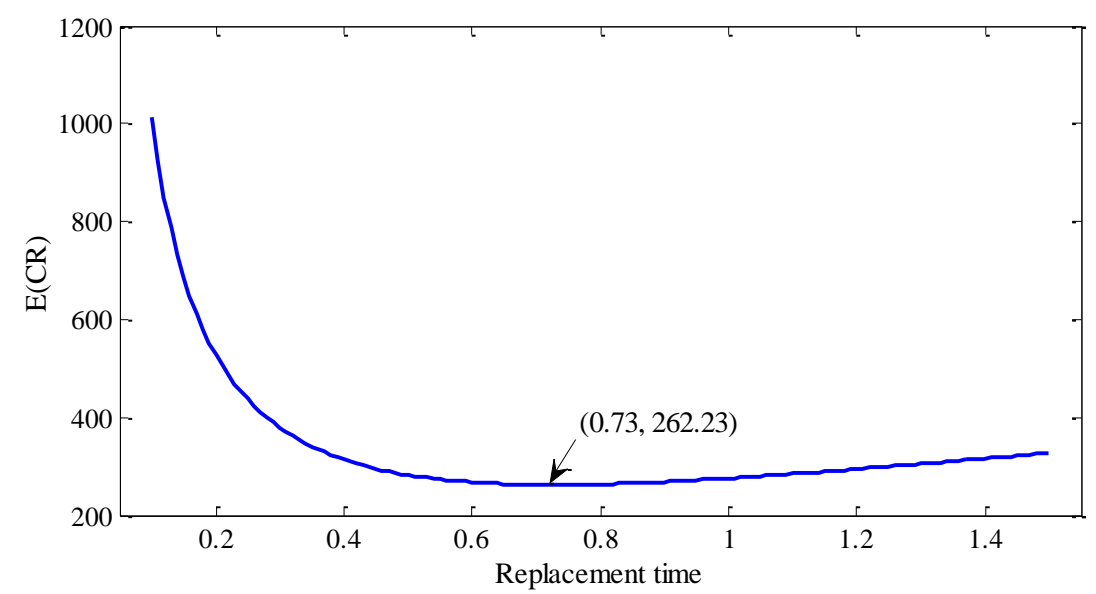

Fig.4 ELRCR for an age-based maintenance policy.

\section{Summary and final remarks}

This paper considered a single-unit system subject to two failure modes, where one failure mode can be modeled by a two-stage delay-time model and the other by a traditional hard failure. For practical systems that consist of multiple failure modes, these failure modes with delay-time could be aggregated as one mode, whilst those failure modes that do not have any detectable defective states before failure could be aggregated as the other mode. Periodic inspections were conducted to detect the possible defective state of the system, and preventive replacements were implemented to mitigate the failure caused by the catastrophic failure mode. We formulated this 
maintenance model and studied the impact of the inspection interval and preventive replacement time on the system performance. Our initial model was then further extended to account for imperfect inspections. The optimal maintenance strategy was investigated and illustrated through a case study of peristaltic infusion pump.

As a direction for future study, the two-stage failure process in this paper can be extended to a three-stage failure process to enable more accurate modeling. Additionally, since the real-world applications generally do not function over an infinite time horizon, the model could be adapted to a finite interval. The dependence between the two failure modes could also be considered in the future. Moreover, failure-inducing inspection is another potential extension, which can be used for multi-component systems.

\section{Acknowledgements}

The research is supported by the NSFC under grant number 71671016 and 71231001, and by the Fundamental Research Funds for the Central Universities of China FRF-BR-15-001B.

\section{Appendix A: Proof of Proposition 1}

It is straightforward to have the derivative of $g(T, n)$ with respect to $n$ as

$$
\begin{aligned}
& \Delta_{n} g(T, n)=g(T, n+1)-g(T, n) \\
& =\int_{n T}^{(n+1) T} R_{3}(t)\left(R_{1}(t)+\int_{n T}^{t} f_{1}(\tau) R_{2}(t-\tau) d \tau\right) d t>0
\end{aligned}
$$

On the other hand, 


$$
\begin{aligned}
& g(T, n)=\int_{0}^{n T} R_{1}(t) R_{3}(t) d t \\
& +\sum_{k=1}^{n} \int_{(k-1) T}^{k T} R_{3}(t) \int_{(k-1) T}^{t} f_{1}(\tau) R_{2}(t-\tau) d \tau d t \\
& <\int_{0}^{n T} R_{1}(t) R_{3}(t) d t \\
& +\sum_{k=1}^{n} \int_{(k-1) T}^{k T} R_{3}(t)\left(R_{1}((k-1) T)-R_{1}(t)\right) d t \\
& =\sum_{k=1}^{n} R_{1}((k-1) T) \int_{(k-1) T}^{k T} R_{3}(t) d t<\int_{0}^{n T} R_{3}(t) d t
\end{aligned}
$$

\section{Appendix B: Proof of Proposition 2}

The derivative of $h(T, n)$ with respect to $n$ can be obtained as

$$
\begin{aligned}
& \Delta_{n} h(T, n)=h(T, n+1)-h(T, n) \\
& =C_{I} R_{3}(n T)\left(R_{1}(n T)+\int_{(n-1) T}^{n T} f_{1}(t) R_{2}(n T-t) d t\right) \\
& +\left(C_{F}-C_{R}\right)\left(\begin{array}{c}
R_{1}(n T) R_{3}(n T)-R_{1}((n+1) T) R_{3}((n+1) T) \\
-R_{3}((n+1) T) \int_{n T}^{(n+1) T} f_{1}(t) R_{2}((n+1) T-t) d t
\end{array}\right)>0
\end{aligned}
$$

Meanwhile, we have

$$
\begin{aligned}
& h(T, n)=C_{I} \sum_{k=1}^{n-1} R_{3}(k T)\left(R_{1}(k T)+\int_{(k-1) T}^{k T} f_{1}(t) R_{2}(k T-t) d t\right) \\
& +C_{R}\left(R_{1}(n T) R_{3}(n T)+\sum_{k=1}^{n} R_{3}(k T) \int_{(k-1) T}^{k T} f_{1}(t) R_{2}(k T-t) d t\right) \\
& +C_{F}\left(1-R_{1}(n T) R_{3}(n T)-\sum_{k=1}^{n} R_{3}(k T) \int_{(k-1) T}^{k T} f_{1}(t) R_{2}(k T-t) d t\right) \\
& <C_{I} \sum_{k=1}^{n-1} R_{3}(k T) R_{1}((k-1) T)+C_{F}<\frac{C_{I}}{T} \int_{0}^{(n-1) T} R_{3}(t) d t+C_{F}
\end{aligned}
$$

Appendix C: Maintenance model with arbitrary preventive replacement interval

Let the preventive replacement period $T_{R}$ be an arbitrary value where $T_{R} \geq T$. 
As the system has to be replaced by time $T_{R}$, the maximum number of inspections is $\left\lfloor T_{R} / T\right\rfloor$. A renewal cycle ends with either preventive replacement or corrective replacement, and we now consider all the possible scenarios this entails.

(1) A defective state is discovered at the $k$ th inspection $\left(k=1,2, \ldots,\left\lfloor T_{r} / T\right\rfloor\right)$. In this case, we have $N_{d}=k-\left\lfloor X_{1} / T\right\rfloor$. The occurrence probability of this scenario can be readily obtained by Eq. (9), with the constraint that $k$ is limited as $k=1,2, \ldots,\left\lfloor T_{r} / T\right\rfloor$. (2) The system is replaced by time $T_{R}$ if no failure occurs and no defective state is detected before $T_{R}$. If the system is in the defective state but has not been identified by inspections, we have $N_{d}>\left\lfloor T_{R} / T\right\rfloor-\left\lfloor X_{1} / T\right\rfloor$. The probability of preventive replacement at time $T_{R}$ can be obtained as

$$
\begin{aligned}
& P\left(T_{c}=T_{R}\right)=\operatorname{Pr}\left\{X_{3}>T_{R}\right\} \\
& \cdot \operatorname{Pr}\left\{\left(X_{1}+X_{2}>T_{R}, X_{1}<T_{R}, N_{d}>\left\lfloor T_{r} / T\right\rfloor-\left\lfloor X_{1} / T\right\rfloor\right) \cup\left(X_{1}>T_{R}\right)\right\} \\
& =R_{3}\left(T_{R}\right)\left(\int_{0}^{T_{R}} f_{1}(t) R_{2}\left(T_{R}-t\right)(1-\beta)^{\left\lfloor T_{R} / T\right\rfloor\lfloor t / T\rfloor} d t+R_{1}\left(T_{R}\right)\right) .
\end{aligned}
$$

(3) A failure occurs if no defective state is discovered and no preventive replacement is implemented. The PDF that a failure occurs by time $t\left(t<T_{R}\right)$ is expressed as 


$$
\begin{aligned}
& f_{T_{f}}(t)=R_{3}(t) \sum_{j=1}^{\lfloor t / T\rfloor} \int_{(j-1) T}^{j T} f_{1}(\tau) f_{2}(t-\tau)(1-\beta)^{\lfloor t / T\rfloor j+1} d \tau \\
& \quad+R_{3}(t) \int_{\lfloor t / T] T}^{t} f_{1}(\tau) f_{2}(t-\tau) d \tau \\
& \quad+f_{3}(t) \sum_{j=1}^{\lfloor t / T\rfloor} \int_{(j-1) T}^{j T} f_{1}(\tau) R_{2}(t-\tau)(1-\beta)^{\lfloor t / T\rfloor-j+1} d \tau \\
& \quad+f_{3}(t) \int_{\lfloor t / T\rfloor T}^{t} f_{1}(\tau) R_{2}(t-\tau) d \tau \\
& \quad+f_{3}(t) R_{1}(t) \\
& \quad=R_{3}(t) \int_{0}^{t} f_{1}(\tau) f_{2}(t-\tau)(1-\beta)^{\lfloor t / T\rfloor\lfloor\tau / T\rfloor} d \tau \\
& \quad+f_{3}(t) \int_{0}^{t} f_{1}(\tau) R_{2}(t-\tau)(1-\beta)^{\lfloor t / T\rfloor-\lfloor\tau / T\rfloor} d \tau \\
& \quad+f_{3}(t) R_{1}(t) .
\end{aligned}
$$

Accordingly, the expected length of a renewal cycle is

$$
\begin{aligned}
& E\left(T_{c}\right)=\sum_{k=1}^{\left\lfloor T_{R} / T\right\rfloor} k T P\left(T_{c}=k T\right)+T_{R} P\left(T_{c}=T_{R}\right)+\int_{0}^{T_{R}} t f_{T_{f}}(t) d t \\
& =\sum_{k=1}^{\left\lfloor T_{R} / T\right\rfloor} k T R_{3}(k T) \int_{0}^{k T} f_{1}(t) R_{2}(k T-t)(1-\beta)^{k-\lfloor t / T\rfloor-1} \beta d t \\
& +T_{R} R_{3}\left(T_{R}\right)\left(\int_{0}^{T_{R}} f_{1}(t) R_{2}\left(T_{R}-t\right)(1-\beta)^{\left\lfloor T_{R} / T\right\rfloor\lfloor t / T\rfloor} d t+R_{1}\left(T_{R}\right)\right) \\
& +\int_{0}^{T_{R}} t\left(\begin{array}{l}
R_{3}(t) \int_{0}^{t} f_{1}(\tau) f_{2}(t-\tau)(1-\beta)^{\lfloor t / T\rfloor\lfloor\tau / T\rfloor} d \tau \\
+f_{3}(t) \int_{0}^{t} f_{1}(\tau) R_{2}(t-\tau)(1-\beta)^{\lfloor t / T\rfloor\lfloor\tau / T\rfloor} d \tau \\
+f_{3}(t) R_{1}(t)
\end{array}\right) d t .
\end{aligned}
$$

The expected cost in a renewal cycle is

$$
\begin{aligned}
& E\left(C_{C}\right)=\sum_{k=1}^{\left\lfloor T_{R} / T\right\rfloor}\left(k C_{I}+C_{R}\right) R_{3}(k T) \int_{0}^{k T} f_{1}(t) R_{2}(k T-t)(1-\beta)^{k-\lfloor t / T\rfloor-1} \beta d t \\
& +\left(\left\lfloor T_{R} / T\right\rfloor C_{I}+C_{R}\right) R_{3}\left(T_{r}\right)\left(\int_{0}^{T_{R}} f_{1}(t) R_{2}\left(T_{R}-t\right)(1-\beta)^{\left\lfloor T_{R} / T\right\rfloor\lfloor t / T\rfloor} d t+R_{1}\left(T_{R}\right)\right) \\
& +\int_{0}^{T_{R}}\left(\lfloor t / T\rfloor C_{I}+C_{F}\right)\left(\begin{array}{l}
R_{3}(t) \int_{0}^{t} f_{1}(\tau) f_{2}(t-\tau)(1-\beta)^{\lfloor t / T\rfloor\lfloor\tau / T\rfloor} d \tau \\
+f_{3}(t) \int_{0}^{t} f_{1}(\tau) R_{2}(t-\tau)(1-\beta)^{\lfloor t / T\rfloor\lfloor\tau / T\rfloor} d \tau \\
+f_{3}(t) R_{1}(t)
\end{array}\right) d t .
\end{aligned}
$$


Appendix D: Maintenance model with time dependent detection probability

If the inspection accuracy is dependent on the time from the initial point of defective stage to the time of inspection, denoted as $\beta(t)$, we can obtain the maintenance cost and length in a similar way as in Section 4. The probabilities of the renewal from inspections and failures are expressed in the following equations.

$$
\begin{aligned}
P\left(T_{c}=k T\right)= & R_{3}(k T) \int_{0}^{k T} f_{1}(t) R_{2}(k T-t) \prod_{j=1}^{k-\lfloor t / T\rfloor-1}(1-\beta(j T+\lfloor t / T\rfloor T-t)) \beta(k T-t) d t, \\
P\left(T_{c}=n T\right)= & R_{3}(n T)\left(\int_{0}^{n T} f_{1}(t) R_{2}(n T-t) \prod_{j=1}^{n-\lfloor t / T\rfloor-1}(1-\beta(j T+\lfloor t / T\rfloor T-t)) d t+R_{1}(n T)\right), \\
f_{T_{f}}^{k}(t)= & R_{3}(t) \sum_{i=1}^{k-1} \int_{(i-1) T}^{i T} f_{1}(\tau) f_{2}(t-\tau) \prod_{j=1}^{k-1-\lfloor\tau / T\rfloor}(1-\beta(j T+\lfloor\tau / T\rfloor T-\tau)) d \tau \\
& +R_{3}(t) \int_{(k-1) T}^{t} f_{1}(\tau) f_{2}(t-\tau) d \tau \\
& +f_{3}(t) \sum_{i=1}^{k-1} \int_{(i-1) T}^{i T} f_{1}(\tau) R_{2}(t-\tau) \prod_{j=1}^{k-1-\lfloor\tau / T\rfloor}(1-\beta(j T+\lfloor t / T\rfloor T-t)) d \tau \\
& +f_{3}(t)\left(R_{1}((k-1) T)-\int_{(k-1) T}^{t} f_{1}(\tau) F_{2}(t-\tau) d \tau\right)
\end{aligned}
$$

Based on the above equations, the expected length and cost of a renewal cycle can be readily obtained.

\section{References}

Aven, T., Castro, I.T., 2009. A delay-time model with safety constraint. Reliability Engineering \& System Safety, 94(2), 261-267.

Berrade, M.D., Cavalcante, C.A.V., Scarf, P.A., 2012. Maintenance scheduling of a protection system subject to imperfect inspection and replacement. European Journal of Operational Research, 218(3), 716-725.

Berrade, M.D., Cavalcante, C.A.V., Scarf, P.A., 2013. Modelling imperfect inspection over a finite horizon. Reliability Engineering \& System Safety, 111,18-29.

Biswajit, S., Saren, S., 2016. Product inspection policy for an imperfect production system with inspection errors and warranty cost. European Journal of Operational Research, 248(1), 263-271. 
Christer, A., 1976. Innovative Decision Making. Presented at the Proc. NATO conference on the role and effectiveness of theories of decision in practice.

Christer, A.H., 1999. Developments in delay time analysis for modelling plant maintenance. Journal of the Operational Research Society, 50(11), 1120-1137.

Christer, A.H., Lee, C., 2000. Refining the delay-time-based PM inspection model with non-negligible system downtime estimates of the expected number of failures. International Journal of Production Economics, 67(1), 77-85.

Dieulle, L., Bérenguer, C., Grall, A., Roussignol, M., 2003. Sequential condition-based maintenance scheduling for a deteriorating system. European Journal of Operational Research, 150(2), 451-461.

Do, P., Vu, H. C., Barros, A., \& Bérenguer, C., 2015. Maintenance grouping for multicomponent systems with availability constraints and limited maintenance teams. Reliability Engineering \& System Safety, 142, 56-67.

Ferreira, R.J.P., de Almeida, A.T., Cavalcante, C.A.V., 2009. A multi-criteria decision model to determine inspection intervals of condition monitoring based on delay time analysis. Reliability Engineering \& System Safety, 94(5), 905-912.

Flage, R., 2014. A delay time model with imperfect and failure-inducing inspections. Reliability Engineering \& System Safety, 124, 1-12.

Golmakani, H.R., Moakedi, H., 2012. Optimal non-periodic inspection scheme for a multi-component repairable system using A* search algorithm. Computers \& Industrial Engineering, 63(4), 1038-1047.

Liu, X., Li, J., Al-Khalifa, K.N., Hamouda, A.S., Coit, D.W., Elsayed, E.A., 2013. Condition-based maintenance for continuously monitored degrading systems with multiple failure modes. IIE Transactions, 45(4), 422-435.

Liu, B., Xie, M., \& Kuo, W., 2016a. Reliability modeling and preventive maintenance of load-sharing systems with degrading components. IIE Transactions, 48(8), 699-709. Liu, B., Xie, M., Xu, Z., \& Kuo, W., 2016b. An imperfect maintenance policy for mission-oriented systems subject to degradation and external shocks. Computers \& Industrial Engineering, 102, 21-32.

Mendes, A.A., Coit, D.W., Ribeiro, J.L.D., 2014. Establishment of the optimal time interval between periodic inspections for redundant systems. Reliability Engineering \& System Safety, 131, 148-165.

Michele, C., Martini, F., Zio, E., 2015. Genetic algorithms for condition-based maintenance optimization under uncertainty. European Journal of Operational Research, 244, 611-623.

Mohammadi, B., Taleizadeh, A.A., Noorossana, R., Samimi, H., 2015. Optimizing integrated manufacturing and products inspection policy for deteriorating manufacturing system with imperfect inspection. Journal of Manufacturing Systems, 37(1), 299-315.

Nakagawa, T., Mizutani, S., Chen, M., 2010. A summary of periodic and random 
inspection policies. Reliability Engineering \& System Safety, 95(8), 906-911.

Nguyen, K. A., Do, P., \& Grall, A., 2015. Multi-level predictive maintenance for multicomponent systems. Reliability Engineering \& System Safety, 144, 83-94.

Park, J., Seager, T.P., Rao, P.S.C., Convertino, M., Linkov, I., 2013. Integrating risk and resilience approaches to catastrophe management in engineering systems. Risk Analysis, 33(3), 356-367.

Peng, H., Feng, Q., Coit, D.W., 2010. Reliability and maintenance modeling for systems subject to multiple dependent competing failure processes. IIE Transactions, 43(1), 12 22.

Phan, D.T., Zhu, Y, 2015. Multi-stage optimization for periodic inspection planning of geo-distributed infrastructure systems. European Journal of Operational Research, 245(3), 797-804.

Sahraoui, Y., Khelif, R., Chateauneuf, A., 2013. Maintenance planning under imperfect inspections of corroded pipelines. International Journal of Pressure Vessels and Piping, 104, 76-82.

Sheils, E., O’Connor, A., Breysse, D., Schoefs, F., Yotte, S., 2010. Development of a two-stage inspection process for the assessment of deteriorating infrastructure. Reliability Engineering \& System Safety, 95(3), 182-194.

Taghipour, S., Banjevic, D., 2012. Optimum inspection interval for a system under periodic and opportunistic inspections. IIE Transactions, 44(11), 932-948.

Wang, W., 1992. Modelling condition monitoring inspection using the delay-time concept (Doctoral dissertation, University of Salford, UK).

Wang, W., 2009. An inspection model for a process with two types of inspections and repairs. Reliability Engineering \& System Safety, 94(2), 526-533.

Wang, W., 2011. An inspection model based on a three-stage failure process. Reliability Engineering \& System Safety, 96(7), 838-848.

Wang, W., 2012. An overview of the recent advances in delay-time-based maintenance modelling. Reliability Engineering \& System Safety, 106, 165-178.

Wang, W., Wu, S., 2014. An inspection model subject to small stoppages and hard failures. Quality Technology and Quantitative Management, 11(3), 255-264.

Williams, G.B., Hirani, R.S., 1997. A delay time multi-level on-condition preventive maintenance inspection model based on constant base interval risk - when inspection detects pending failure. International Journal of Machine Tools and Manufacture, 37(6), 823-836.

Wu, S., Chen, Y., Wu, Q., Wang, Z., 2016. Linking component importance to optimisation of preventive maintenance policy. Reliability Engineering \& System Safety, 146, 26-32.

Wu, S., Coolen, F. P., \& Liu, B., 2017. Optimisation of maintenance policy under parameter uncertainty using portfolio theory. IISE Transactions, 49(7), 711-721.

Zhao, X., Nakagawa, T., 2015. Optimal periodic and random inspections with first, last 
and overtime policies. International Journal of Systems Science, 46(9), 1648-1660. Zhao, F., Wang, W., Peng, R., 2015. Delay-time-based preventive maintenance modelling for a production plant: a case study in a steel mill. Journal of the Operational Research Society, 66, 2015-2024. 